\title{
Semi-analytical solutions for the diffusive logistic equation with mixed instantaneous and delayed density dependence
}

H.Y. Alfifi ${ }^{1 *}$

"Correspondence:

hyalfifi@iau.edu.sa

1 Department of Basic Sciences,

College of Education, Imam

Abdulrahman Bin Faisal University,

Dammam, Saudi Arabia

\section{Springer}

\begin{abstract}
In this work, the semi-analytical solution is studied for the diffusive logistic equation with both mixed instantaneous and delayed density. The domain of reaction-diffusion in one dimension is shown. Delay partial differential equation is approximated with a delay ordinary differential equation system by using the Galerkin technique method. Steady-state solutions and stability analysis as well as bifurcation diagrams are derived. The effect of diffusion parameter and delay values is comprehensively studied; as a result, both parameters can destabilize or stabilize the model. We obtained that the decrease in values of the Hopf bifurcations for growth rate is associated with an increase in delay values, whereas the diffusion parameter is increased. Furthermore, comparisons between the numerical simulations and semi-analytical results present a good agreement for all examples and figures of the Hopf bifurcations. Examples of limit cycle and phase-plane map are plotted to confirm the benefits and accuracy of semi-analytical solutions result. For periodic solutions, an asymptotic method is studied after the Hopf bifurcation point for both one- and two-term semi-analytical systems.
\end{abstract}

MSC: 35K57; 34C05; 35B32; 34K18; 35B10; 35R10; 92D25; 92D40

Keywords: Semi-analytical solution; Logistic equation; Stability analysis; Hopf bifurcation; Limit cycle; Reaction-diffusion; Delay equation

\section{Introduction}

Reaction-diffusion phenomenon with time delays has been incorporated into many fields of biological applications. These applications have explained a number of practical applications in our everyday life by using partial differential equations (PDEs), for instance, in population ecology $[15,17,20,30,31]$, animals $[2,4,26]$, cell $[5,19,22,25,33]$, chemicals $[1,3,10]$, and heat and mass transfer $[13,27]$. This model can introduce instability, via a Hopf bifurcation, with the subsequent development of limit cycles. The oscillatory phenomena (periodic solutions) have been shown by utilizing a continuous well-stirred tank reactor (CSTR). CSTR has a significant importance to show good outcomes with theoretical and experimental investigations: for more details, see $[4,5,8]$ and the references therein.

(c) The Author(s) 2020. This article is licensed under a Creative Commons Attribution 4.0 International License, which permits use sharing, adaptation, distribution and reproduction in any medium or format, as long as you give appropriate credit to the original author(s) and the source, provide a link to the Creative Commons licence, and indicate if changes were made. The images or other third party material in this article are included in the article's Creative Commons licence, unless indicated otherwise in a credit line to the material. If material is not included in the article's Creative Commons licence and your intended use is not permitted by statutory regulation or exceeds the permitted use, you will need to obtain permission directly from the copyright holder. To view a copy of this licence, visit http://creativecommons.org/licenses/by/4.0/. 
The delay logistic equation with diffusion has been discussed extensively. [32] considered a diffusive logistic equation with delays. The stability analysis and Hopf points were explored by the characteristic equation. The technique of normal form along with the theory of manifold center was used to find the stability analysis and the direction of the Hopf bifurcation. The researchers provided some numerical examples to confirm their theoretical results. Furthermore, [6] developed a lower-order semi-analytical system for the delay logistic equation with feedback term. Galerkin's method was explored to show delay systems of ordinary differential equations (ODE). The Hopf bifurcation curves' maps and steady-state solutions were discussed. The non-smooth ODE system was constructed, which showed that increasing the delay values in the equation is destabilizing, whilst increasing the feedback delay stabilizes the model. The semi-analytical solutions represent a novel way of predicting the stability of a non-smooth PDE model. In addition, Alfifi et al. [7] discussed the class of generalized logistic delay PDEs in a similar way, these classes are distributed and point delays. Galerkin's method of approximation was utilized to discretize the results. The semi-analytical outcomes were comprehensively developed. A great comparison was achieved through using semi-analytical methods. [12] investigated stability analysis and Hopf bifurcation points in a diffusive logistic population that has a nonlocal effect of delay. [11] considered the Hopf bifurcation of the delay diffusive logistic equation with the introduced Dirichlet condition for boundaries. [16] discussed delay-diffusion equations with a small value of delay parameter. They derived that all results were asymptotic to the set of equilibrium (similar to an equation without delay) as the time approaches infinity.

Semi-analytical methods have been used to discuss many delay systems with reactiondiffusion phenomenon, such as delay logistic equations [6,7], predator-prey model [2], viral infection system [5], pellet systems [24], the Belousov-Zhabotinsky (BZ) reaction [9], the Brusselator model [3], the reversible Selkov model [1], the equation of Nicholsons blowflies [8], and the limited food model [4]. The outcomes for all papers that used this method revealed an excellent agreement between semi-analytical ODEs outcomes and the numerical solutions pertain to PDEs equations.

This paper has various objectives. Galerkin's method provides a useful technique to accurately predict calculation for this system. So we discuss the effect of diffusion and delay values of this system and also give illustrations of how the Hopf bifurcation points can be calculated, discuss the stability of the system. In addition, the objective is to find an asymptotic analysis close the Hopf bifurcation points. Thus, this paper has been organized as follows: Sect. 2 shows a non-dimensional delay diffusive system and the Dirichlet boundary specification. In Sect. 3 we demonstrate the application of Galerkin's method, which helps to find the ODEs system. Section 4 discusses a stability analysis, constructing bifurcation diagrams, and performing comparisons of the semi-analytical and numerical results. In Sect. 5, an asymptotic analysis of periodic result close to the point of Hopf bifurcation is established in detail.

\section{The semi-analytical system}

The non-linear PDE logistic equation growth with both instantaneous and delayed density dependence is written as follows:

$$
u_{t}=D u_{x x}+\mu u(t)[1-\alpha u(t)-\beta u(t-\tau)] .
$$


In this equation, $u$ refers to the density of population at location $x$ and time $t . \mu$ describes the proliferation rate (growth rate), $\tau$ represents the delay term, and $D$ refers to the diffusion coefficient of the logistic equation. The parameter $\alpha$ stands for the portions of instantaneous growth rate and $\beta$ represents delay dependence of the growth rate. For easiness of notation, equation (1) is rescaled by using $u^{*}(x, t)=u(x, t \tau)$ and then dropping the asterisks. Therefore, the non-dimensional diffusive system with delay and the Dirichlet boundary specification is written as follows:

$$
\begin{aligned}
& u_{t}=D \tau u_{x x}+\mu \tau u(t)[1-\alpha u(t)-\beta u(t-1)], \\
& u(x, t)=0, \quad \text { at } x= \pm 1, \quad u(x, t)=u_{a}, \quad-\tau<t \leq 0 .
\end{aligned}
$$

Here, zero flux boundary condition is obtained in the middle point of domain. So at the center of domain, the solution has a symmetrical pattern. Moreover, $u_{a}$ is considered to be a positive constant initial population, when time ranges in $(-\tau, 0)$. Note that the parameter $u_{a}=0.5$ is used in all figures and examples in this paper. The fourth-order Runge-Kutta technique $[8,24]$ is utilized to compute the solution of a delay ODEs system, while the finite-difference approximation [5, 7] is used for the numerical results of a single PDE equation. The spatial and temporal discretizations solved in this paper will be $\Delta t=5 \times 10^{-3}$ and $\Delta x=0.01$. The inaccuracy proportion is examined to find the difference between the values of semi-analytical results and the values of the numerical solutions which are derived by dividing by the precise estimate times of 100 .

\section{The Galerkin method technique}

Galerkin's technique utilizes orthogonality of a basis functions set, so to transform PDE into coupled sets of ODEs. The method can be thought of most usefully as a temporalspatial separation. This technique considers a spatial form of the profile concentration, which is described in $[5,7,23,24]$. Galerkin's method indicates an analytical technique, which utilizes the orthogonality of rudimentary roles set, so to consider the delay ODEs model from the PDE equation. Many authors have used this technique in different areas and models, for example, see $[6-8,23]$. Here, the following expression is a trial equation, which was used:

$$
u(x, t)=u_{1}(t) \cos \left(\frac{\pi x}{2}\right)+u_{2}(t) \cos \left(\frac{3 \pi x}{2}\right) .
$$

The trial function is established, in which $u=\sum u_{i}$ refers to the population density $u$ at the center of domain. Trial equation (3) subtends the boundary conditions in PDE equation (1). The free values of parameters in this equation are then calculated by the evaluation of mean values of the PDE equation, which is weighted by two functions. These functions are $\cos \left(\frac{1}{2} \pi x\right)$ and $\cos \left(\frac{3}{2} \pi x\right)$. Hence, a system of ODEs is considered as follows:

$$
\begin{aligned}
\frac{d u_{1}}{d t}= & -\frac{\pi^{2}}{4} D \tau u_{1}+\tau \mu u_{1}-\frac{16}{15 \pi} \alpha \tau \mu u_{1} u_{2}-\frac{72}{35 \pi} \alpha \tau \mu u_{2}^{2}-\frac{8}{3 \pi} \beta \tau \mu u_{1} u_{1 d} \\
& -\frac{8}{15 \pi} \beta \tau \mu u_{1} u_{2 d}-\frac{8}{15 \pi} \beta \tau \mu u_{2} u_{1 d}-\frac{72}{35 \pi} \beta \tau \mu u_{2} u_{2 d}-\frac{8}{3 \pi} \alpha \tau \mu u_{1}^{2}, \\
\frac{d u_{2}}{d t}= & -\frac{9 \pi^{2}}{4} D \tau u_{2}+\tau \mu u_{2}-\frac{144}{35 \pi} \alpha \tau \mu u_{1} u_{2}+\frac{8}{9 \pi} \alpha \tau \mu u_{2}^{2}-\frac{8}{15 \pi} \beta \tau \mu u_{1} u_{1 d}
\end{aligned}
$$




$$
\begin{gathered}
\quad-\frac{72}{35 \pi} \beta \tau \mu u_{1} u_{2 d}-\frac{72}{35 \pi} \beta \tau \mu u_{2} u_{1 d}+\frac{8}{9 \pi} \beta \tau \mu u_{2} u_{2 d}-\frac{8}{15 \pi} \alpha \tau \mu u_{1}^{2}, \\
u_{1 d}=u_{1}(x, t-1) u_{2 d}=u_{2}(x, t-1) .
\end{gathered}
$$

The series in this case are abbreviated after two-term expansion. The reason for that is that the two-term solution gets enough accuracy without extreme swell of expression. In particular, the equation with one term is presented by setting $u_{2}=0$ in equation (4).

\section{Bifurcation diagrams and stability analysis}

\subsection{Methodology and theoretical framework}

This part examines methodologies utilized to provide a map of Hopf bifurcations for the semi-analytical model of (4). A Hopf bifurcation signifies the appearance of limit cycles at the neighborhood of the steady-state stability changes from stable to unstable solutions as a consequence of a conjugated pair of eigenvalue values, which passes over the imaginary axis $[7,29]$. There are many books about the stability analysis and dynamical systems described for the ODEs system [14, 18]. In our case, the Hopf bifurcation map is computed by finding an expansion through the Taylor series constructed for the steady-state values, this can be obtained as the following equation:

$$
u_{1}=u_{1 s}+\epsilon \rho_{1} e^{-z t} \quad \text { and } \quad u_{2}=u_{2 s}+\epsilon \rho_{2} e^{-z t}, \quad \epsilon \ll 1 \text {. }
$$

Hence, equations $u_{1}$ and $u_{2}$ as in (5) are substituted into ODEs model (4). After that, they are linearized about the steady-state values. Then, the Jacobian matrix of eigenvalues develops a small system perturbation, which explains the typical growth rate $z$, by letting $z=i \omega$ in the expression of the characteristic equation, and then by separation of the real part (Real) and the imaginary part of equation (Imag). Thus, the following equations can obtain the Hopf bifurcation points as follows:

$$
\text { Real }=\operatorname{Imag}=\frac{d u_{1}}{d t}=\frac{d u_{2}}{d t}=0
$$

where $\frac{d u_{1}}{d t}$ and $\frac{d u_{2}}{d t}$ stand for the steady-state system of (4).

\subsection{Hopf bifurcation areas}

In this part, semi-analytical maps are developed for a system of equations (4), in which the Hopf bifurcations are found and compared with numerical results. Furthermore, diffusion parameters and the effects of delay in the system are studied by a number of illustrations and figures.

Figure 1 shows a map of two regions with Hopf bifurcation in $\tau$ versus $\mu$ plane. The values used are $D=0.3, \alpha=0.5$, and $\beta=0.7$. The numerical (cross points) and the twoterm (dotted line) semi-analytical results are shown. There are two regions in this figure: the upper part of the curve refers to unstable region, while the down region shows a stable area. It shows that once there is a growth in the delay values $\tau$, then the values of the Hopf bifurcation points for growth rate $\mu$ are decreasing. Furthermore, we found that at small values of delay parameter $\tau$, the Hopf bifurcation points $\mu$ are extremely similar to the case without delay. This behavior is qualitatively conformable with the results in [16]. It obviously can be seen that there is an agreement between two-term result against the 
Figure 1 (color online) Shows the Hopf bifurcation map for $\tau-\mu$. The red cross points represent the solution of the numerical PDE equation and the black dots refer to a solution of the semi-analytical equation with two terms. The positive values used here are $D=0.3, \alpha=0.5$, and $\beta=0.7$

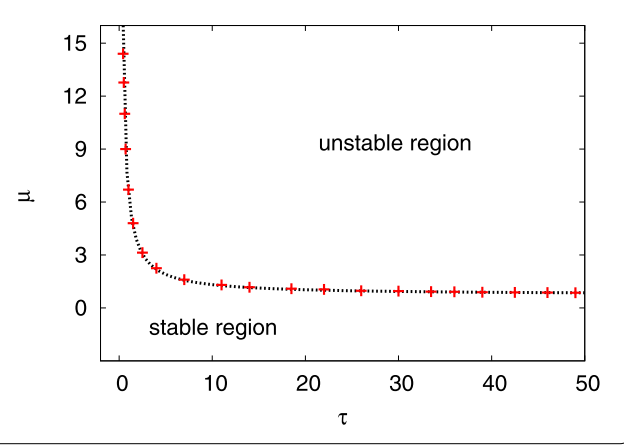

Figure 2 (color online) Displays the Hopf bifurcation regions of the delay values $\tau$ versus the parameters of $\alpha$ and $\beta$. The semi-analytical results with two terms are plotted. The parameters are $D=0.3, \mu=5$, and $\beta=0.7$ in the $\tau-\alpha$ plane (dotted red curve), while $\alpha=0.7$ for the $\tau-\beta$ plane (black dash curve)

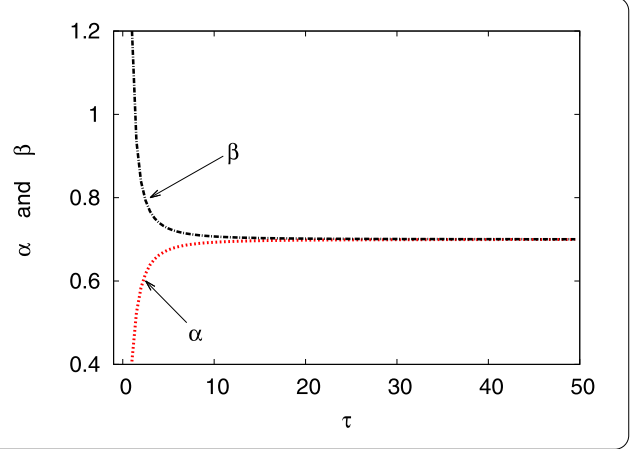

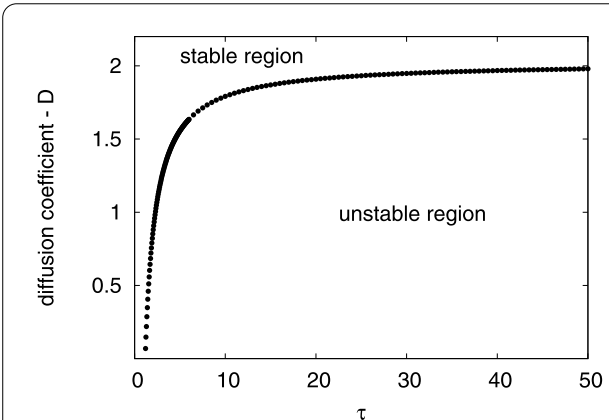

(a)

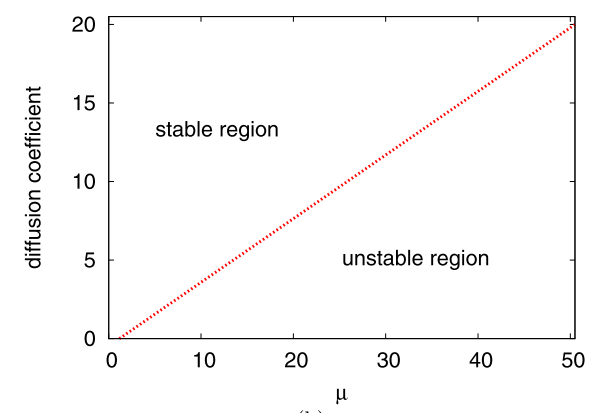

(b)

Figure 3 (color online) (a) Demonstrates the Hopf bifurcation curves in the $\tau-D$ map and $\mu-D$ map for (b). The two-term semi-analytical solution is provided. The parameters used are $\alpha=0.5, \beta=0.7$, and $\mu=5$ for Figure (a), while $\alpha=0.5, \beta=0.7$, and $\tau=5$ for Figure (b)

numerical result, with a very small error between them with less than $1 \%$ for all $\mu$ choices up to 50 .

Figure 2 exhibits Hopf bifurcation curves in the delay value $\tau$ versus portions of instantaneous growth rate $\alpha$ (dotted curve) and delay dependence of the growth rate $\beta$ (dashed curve). The two-term semi-analytical results are obtained. The value of portions of instantaneous growth rate $\alpha$ is increased linearly as delay values increase. However, the curve of value $\beta$ is decreased as value of $\tau$ is increased. It is clear that for a long value of delay parameter $\tau$ the values of $\alpha \simeq \beta$, while $\beta>\alpha$ at a small value of $\tau$, this result is similar to [16].

Figures 3(a) and 3(b) explain the Hopf bifurcation maps for the delay parameter $\tau$ (in the left pane) and the growth rate $\mu$ (in the right pane) against diffusion parameters $D$. 
The parameters are $\alpha=0.5, \beta=0.7$, and $\tau=5$ for Fig. 3(a) and $\alpha=0.5, \beta=0.7$, and $\mu=5$ for Fig. 3(b). The semi-analytical two-term solution is used in each case. In these cases, there are two regions: stable and unstable. Also, the Hopf bifurcation of the proliferation rate $\mu$ and the delay parameter $\tau$ rises as the diffusion coefficients $D$ are increased. The results obtained in Fig. 3(b) agree with the same outcome in [28, 32], so when $\mu \leq D$, zero solution is the global attractor of all non-negative solutions, while $\mu>D$ has a unique positive steady-state result. Therefore, the diffusion coefficient $D$ can play an extremely important role in the system and can indicate the model that is destabilized or stabilized.

Furthermore, the comparisons are provided for the special values as follows: $D=0.3$, $\tau=12, a=0.3$, and $b=0.7$. In our special case, the points of Hopf bifurcation derived are $\mu_{c} \simeq 1.22,1.23$ for both semi-analytical one- and two-term results, while $\mu_{c} \simeq 1.23$ is for the numerical result. The predictions of semi-analytical two-term result agree with the numerical predictions of PDE solution; in addition, there is less than $1 \%$ error with one-term result. The semi-analytical solution gives a reliable prediction of the incidence of Hopf bifurcation points.

\subsection{Bifurcation maps, steady-state and oscillatory solutions}

In this section, the steady-state solutions and the bifurcation maps and periodic solution (limit cycle) are shown. In the bifurcation map, the minimum and maximum amplitudes of limit cycle oscillations and steady-state amplitude are plotted at long time result. In addition, the bifurcation maps are explained in the domain center. Note that the parameters $\alpha=0.5, \beta=0.7$, and $u_{a}=0.5$ are applied in all figures in this part.

Figure 4 represents the behavior of $u$, the population steady-state density, versus value of $\mu$ (proliferation rate), at $x=0$. Semi-analytical models with one and two terms along with numerical solutions are obtained. We let $u_{i}(t)=u_{i d}=u_{i}(t-1)$ in ODEs model (4), which can be reduced to sets of transcendental equations. A unique solution of the steady state is derived for the parameter $u$ (population density). The non-uniform steady-state solution bifurcates from the steady-state uniform solution of $u=0$ at the point of $\mu=0.745$ and increases exponentially as $\mu$ increases before approaching a maximum population density of the numerical solution at $u \simeq 0.8$. There is a good length of agreement among the semianalytical two-term solutions and numerical outcomes, with no more than $1.5 \%$ error for the values of all parameters of growth rate up to $\mu=100$.

Figure 5 provides the bifurcation diagram of the concentration $u$ versus the proliferation rate $\mu$. This figure can show how closely numerical and semi-analytical results are related. The two- and one-term semi-analytical results and the numerical results are plotted. The values used here are $\tau=5$ and $D=0.3$. It was found that steady-state solutions

Figure 4 (color online) The population density in steady state $u$ at the center of domain $x=0$. The figure shows semi-analytical one-term (blue dashed curve) and two-term (red dotted curve) solutions of (4) and the numerical solution (black solid curve) of the PDE

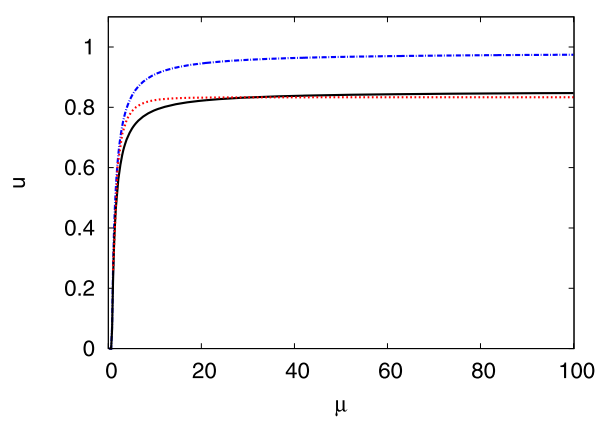


Figure 5 (color online) Bifurcation map of the population density $u$ against the growth rate $\mu$. The two-term semi-analytical result (dotted red curve), one-term semi-analytical result (dashed blue curve), and the numerical PDE solution (solid black curve) are plotted. The values of parameters are $\tau=5$ and $D=0.3$

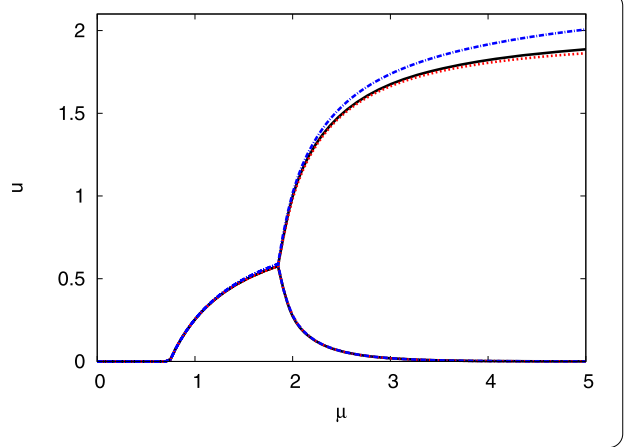

Figure 6 (color online) Bifurcation diagram of the population density $u$ versus $\mu$ with three different values of diffusion parameters $D$. The semi-analytical two-term solutions are plotted with $D=0.1$ (displayed as blue dashed curve), $D=0.3$ (shown as red dotted curve), and $D=0.5$ (black solid curve)

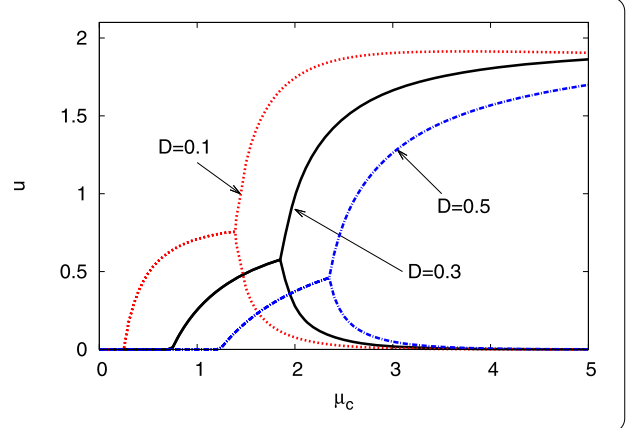

are stable when $\mu<\mu_{c} \simeq 1.90$. After this point, there are periodic solutions, the so-called supercritical Hopf bifurcations begin. Here, the Hopf bifurcation points are $\mu_{c}=1.897$ and 1.901, respectively. The Hopf bifurcation point for the numerical solution is at the value of $\mu_{c}=1.903$. This figure shows that when $\mu$ increases, the maximum amplitude at oscillations is growing, while in the minimum points, it is decreasing. Therefore, the predictions applying to the two semi-analytical results agree with the predictions obtained from numerical results, with less than $1 \%$ error for all values selected of $\mu$ in the domain.

Figure 6 shows the bifurcation map of the population density against proliferation rate $\mu$ with three different cases of diffusion parameters $D=0.1$ (dashed curve), $D=0.3$ (dotted curve), and $D=0.5$ (solid curve). The semi-analytical two-term results are obtained for each case. The supercritical Hopf bifurcation solutions are established at $\mu=1.39$ for the case with $D=0.1$ and at $\mu=1.90,2.38$ for the cases with $D=0.3$ and $D=0.5$. It is clearly visible that the effect of increasing diffusion parameters stabilizes the system. This figure confirms the outcome in Fig. 3(b).

Figure 7 shows the bifurcation map in a $\mu-u$ plane. Three different cases of delay values $\tau: \tau=3$ (solid curve), $\tau=5$ (dotted curve), and $\tau=10$ (dashed curve) are shown. The two-term semi-analytical result is plotted. The supercritical Hopf bifurcation solutions are found at $\mu=2.67$ for the case with delay value $\tau=3$ and at $\mu=1.90,1.32$ for the cases when delay values are $\tau=5$ and $\tau=10$, respectively. It can be concluded that the increase in delay parameters destabilizes the system, so in this case the values of Hopf bifurcation points $\mu$ are decreased. This figure confirms the results in Fig. 3(a).

Figure 8(a) exhibits $u$, the population density at $x=0$, versus time $t$, while Fig. 8(b) shows phase-plane maps for $u$. The values used here are $\tau=5$ and $\mu=1.6$, so the solution here is stable for both figures (see stable region provided in Fig. 5). In addition, $\mu_{c}=1.90<\mu_{c}$ and the steady-state solution is formed at $u(0, t)=0.46$ when the time $t$ is large enough. 
Figure 7 (color online) Bifurcation diagram of the growth rate $\mu$ versus population density $u$, with three different values of $\tau: \tau=10$ (blue dashed curve), $\tau=5$ (red dotted curve), and $\tau=3$ (black solid curve). The two-term semi-analytical result is shown

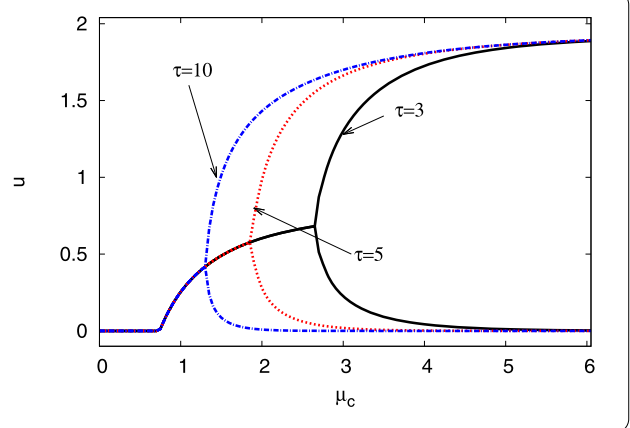

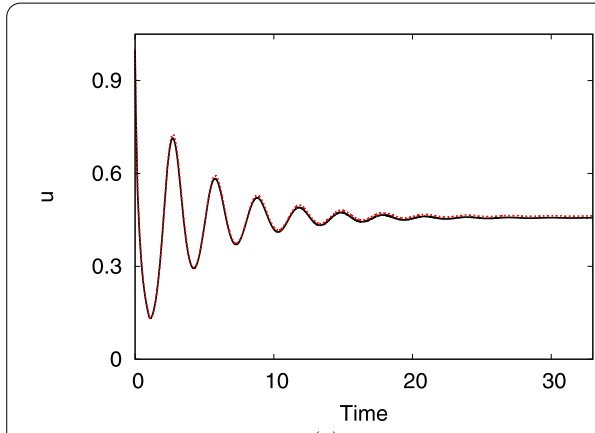

(a)

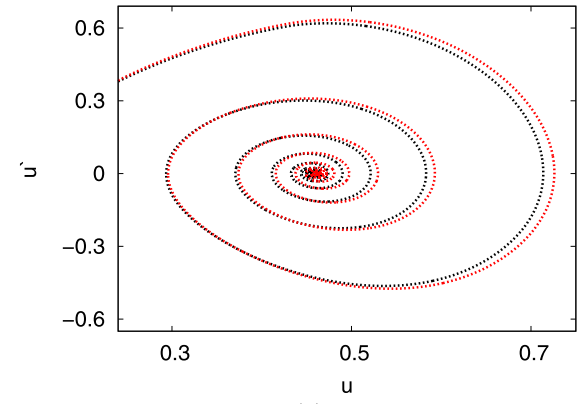

(b)

Figure 8 (color online) Shows phase-plane maps for $u$. The semi-analytical two-term solution (dotted black curve) and the numerical solutions (dotted black curve) are obtained for $\tau=5$ and $\mu=1.6$

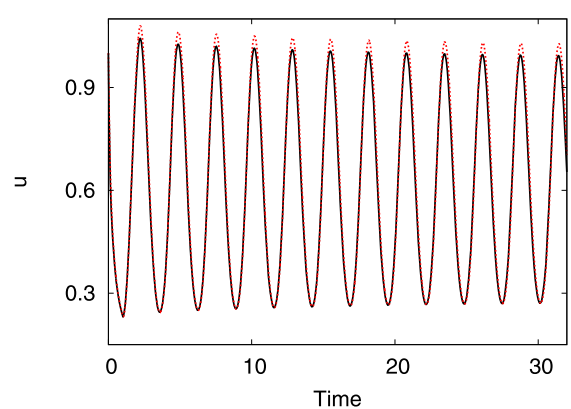

(a)

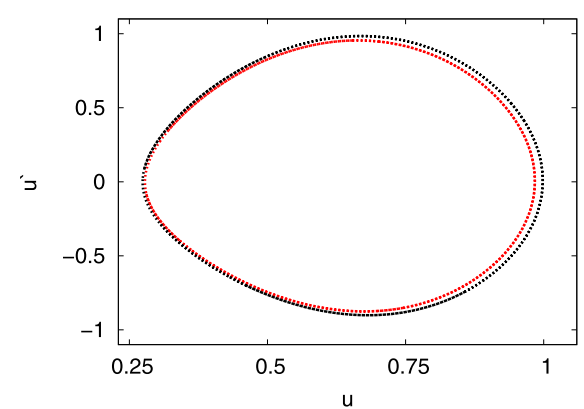

(b)

Figure 9 (color online) Shows phase-plane maps for the population density $u$. The semi-analytical two-term solution shown by black dotted line and the numerical solution represented by red solid line with $\tau=5$ and $\mu=2$ are obtained

The semi-analytical two-term solutions and numerical outcomes are obtained. Here, these results agree with an error rate of more than $1 \%$.

Both Figs. 9(a) and (b) show phase-plane maps for the parameter of population density $u$. The values applied are $\tau=5$ and $\mu=2$ as illustrated from limit cycle occurrence after passing through the point of Hopf bifurcation at $\mu<\mu_{c} \simeq 1.90$, this point is chosen from unstable region in Fig. 5. The scheme of numerical and two-term semi-analytical solution is given. The numerical results are extremely close to the semi-analytical outcomes, with an error rate less than $1.5 \%$. On the other hand, the one-term semi-analytical result is accurate compared to the numerical results of the PDE equation. 
Figure 10 (color online) Phase-plane maps are shown for the semi-analytical two-term solutions with parameter value $\tau=5$ for four different cases of growth rate $\mu: \mu=2$ (red dot curve), $\mu=2.5$ (black dot curve), $\mu=3$ (blue dot curve), and $\mu=3.5$ (green dot curve)

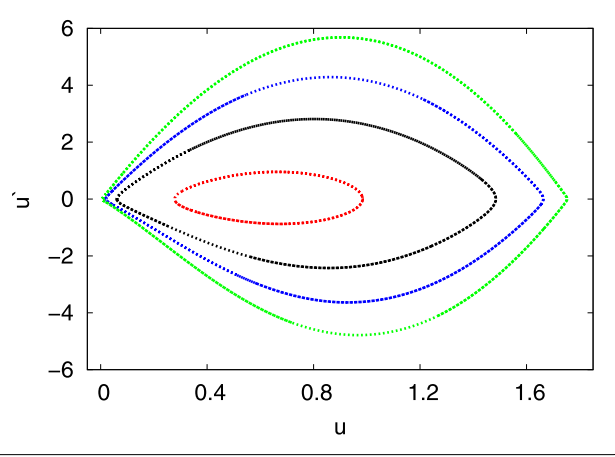

Figure 10 displays the 2-D phase space of the concentrations $u$ with limit cycle for four various values of $\mu: \mu=2$ (red dot curve), $\mu=2.5$ (black dot curve), $\mu=3$ (blue dot curve), and $\mu=3.5$ (green dot curve). The semi-analytical two-term result is plotted. The value of delay parameter is $\tau=5$. This figure shows periodic solutions for higher values of proliferation rate $\mu$ after the point of the Hopf bifurcation. There are many biological models, for example, Nicholson's blowflies model [8], that show a period doubling, which means the solutions are chaotic. However, this did not happen for this system of PDE or ODEs equations. Figure 10 demonstrates that the increase in oscillation amplitudes is associated with the increase in growth rate $\mu$.

\section{Periodic solutions after the Hopf bifurcation point}

In this section, an asymptotic analysis is performed for a periodic solution close to the point of Hopf bifurcation for semi-analytical delay ODEs system and delay PDE equation. We build an asymptotic solution of both semi-analytical systems, which include ODEs with delay, utilizing the method of the Hopf perturbation used in $[8,9,14]$.

In this part, the power series solution is developed near the Hopf bifurcation point in the small oscillation amplitude limits. The mentioned technique extends the outcome result around steady-state results at the point of Hopf bifurcation. Then, it determines the changes applied to bifurcation and frequency values. After that, it uses conditions of solvability, eliminating secular terms; for more details, see $[9,21]$.

\subsection{The one-term semi-analytical solution}

The semi-analytical one-term system is derived by a single equation

$$
\begin{aligned}
& \frac{d u_{1}}{d t}=-\frac{\pi^{2}}{4} D \tau u_{1}+\tau \mu u_{1}-\frac{8}{3 \pi} \alpha \tau \mu u_{1}^{2}-\frac{8}{3 \pi} \beta \tau \mu u_{1} u_{1 d}, \\
& u_{1 d}=u_{1}(x, t-1) .
\end{aligned}
$$

The solution technique for the one-term equation is identical to that in references [8, 9, $14]$. We let a solution with $2 \pi$-periodic for equation (6) to have the following form:

$$
u_{1}(s)=z_{s}+\epsilon z_{1}(s)+\epsilon^{2} z_{2}(s)+\epsilon^{3} z_{3}(s)+\cdots, \quad \text { where } s=w t .
$$

The values of $\epsilon$ and scaled time are the amplitude of limit cycle solutions (periodic oscillations) after the Hopf bifurcation point, which is determined by applying the condition 
of normalization

$$
\epsilon=\frac{1}{2 \pi} \int_{0}^{2 \pi} z(s, \epsilon) e^{-i s} d s
$$

This implies

$$
\frac{1}{2 \pi} \int_{0}^{2 \pi} z_{1}(s) e^{-i s} d s=1, \quad \int_{0}^{2 \pi} z_{i}(s) e^{-i s} d s=0, \quad \forall i \neq 1
$$

After that, the bifurcation value of the growth rate $\mu$ and frequency $\omega$ are defined by applying a power series as follows:

$$
\mu=\mu_{0}+\epsilon^{2} \mu_{2}+\cdots, \quad \omega=\omega_{0}+\epsilon^{2} \omega_{2}+\cdots
$$

Here, $\mu_{0}$ and $\omega_{0}$ are Hopf bifurcation point values. Then, we utilize solvability conditions with the order of 3 for the perturbation analysis $\epsilon$. Therefore, the corrections $\mu_{2}$ and $\omega_{2}$ are shown. Equations (7) and (10) are substituted into one-term equation (6). Therefore, the first three orders of $\epsilon$ are as follows:

$$
\begin{aligned}
\omega_{0} \dot{z_{1}}= & -\frac{\pi^{2}}{4} D \tau z_{1}+\tau \mu_{0} z_{1}-\frac{8 \mu_{0} z_{s} \tau}{3 \pi}\left(2 \alpha z_{1}+\beta z_{1}+\beta z_{1 \tau}\right), \\
\omega_{0} \dot{z_{2}}= & -\frac{\pi^{2}}{4} D \tau z_{2}+\tau \mu_{0} z_{2}+\tau \mu_{2} z_{s}-\frac{8 \tau \mu_{2} z_{s}^{2}}{3 \pi}(\alpha+\beta) \\
& -\frac{8 \tau \mu_{0}}{3 \pi}\left(2 \alpha z_{2} z_{s}+\beta z_{2} z_{s}+\alpha z_{1}^{2}+\beta z_{1} z_{1 \tau}+\beta z_{s} z_{2 \tau}\right), \\
\omega_{0} \dot{z_{3}}= & -\frac{\pi^{2}}{4} D \tau z_{3}+\tau \mu_{0} z_{3}+\tau \mu_{2} z_{1}-\frac{8 \tau \mu_{2} z_{s}}{3 \pi}\left(\alpha z_{1}+\beta z_{1 \tau}\right)-\omega_{2} \dot{z}_{1} \\
& -\frac{8 \tau \mu_{0}}{3 \pi}\left(2 \alpha z_{3} z_{s}+2 \alpha z_{1} z_{2}+\beta z_{3} z_{s}+\beta z_{2} z_{1 \tau}+\beta z_{s} z_{3 \tau}+\beta z_{1} z_{2 \tau}-\beta \omega_{2} z_{s} z_{1 \tau}\right) .
\end{aligned}
$$

Equation (11) admits $2 \pi$-periodic solution of the form

$$
z_{1}(s)=e^{i s}+\text { c.c., }
$$

where the complex conjugate is defined as c.c. Here we can see $z_{1}$ amplitude is selected to meet the normalization condition (9). For $\mathrm{O}\left(\epsilon^{2}\right)$, the result of $z_{2}$ can be written as the form

$$
z_{2}(s)=A e^{2 i s}+\text { c.c. }
$$

Substituting (14) and (15) into (12) gives

$$
\begin{aligned}
2 i A \omega_{0}= & -\frac{\pi^{2}}{4} A \tau D+\tau \mu_{0} A \\
& -\frac{8 \tau \mu_{0}}{3 \pi}\left(2 \alpha A z_{s}+\beta A z_{s}+\alpha+\beta e^{-i \omega_{0} \tau}+A \beta z_{s} e^{-2 i \omega_{0} \tau}\right)+\text { c.c. }
\end{aligned}
$$


as an equation for the complex amplitude $A$. We let $A=A_{r}+i A_{i}$ and separate (16) into real and imaginary parts as follows:

$$
\begin{aligned}
-2 A_{i} \omega_{0}= & -\frac{8 \tau \mu_{0}}{3 \pi}\left[2 \alpha A_{r} z_{s}+\beta A_{r} z_{s}+\alpha+\beta \cos \left(\omega_{0} \tau\right)+A_{r} \beta z_{s} \cos \left(2 \omega_{0} \tau\right)\right] \\
& -\frac{\pi^{2}}{4} A_{r} \tau D+\tau \mu_{0} A_{r} \\
2 A_{r} \omega_{0}= & -\frac{8 \tau \mu_{0}}{3 \pi}\left[2 \alpha A_{i} z_{s}+\beta A_{i} z_{s}-\beta \sin \left(\omega_{0} \tau\right)-A_{i} \beta z_{s} \sin \left(2 \omega_{0} \tau\right)\right] \\
& -\frac{\pi^{2}}{4} A_{i} \tau D+\tau \mu_{0} A_{i}
\end{aligned}
$$

for the two components of $A$. Afterwards we examine (13), the equation for $z_{3}$. Substituting the known first and second order solutions $z_{1}$ and $z_{2}$ gives

$$
\begin{aligned}
\Rightarrow \omega_{0} \dot{z}_{3} & +\frac{\pi^{2}}{4} D \tau z_{3}-\tau \mu_{0} z_{3} \\
& +\frac{8 \tau \mu_{0}}{3 \pi}\left(2 \alpha z_{3} z_{s}+2 \alpha z_{1} z_{2}+\beta z_{3} z_{s}+\beta z_{2} z_{1 \tau}+\beta z_{s} z_{3 \tau}+\beta z_{1} z_{2 \tau}\right) \\
= & \tau \mu_{2} e^{i s}-\frac{8 \tau \mu_{2} z_{s}}{3 \pi}\left(\alpha e^{i s}+\beta e^{i s} e^{-i \omega_{0} \tau}\right)+e^{i s} \frac{8 \tau \mu_{0}}{3 \pi}\left(i \beta \omega_{2} z_{s} e^{-i \omega_{0} \tau}\right) \\
& -\omega_{2} \dot{z_{1}}+\text { c.c. }
\end{aligned}
$$

The solvability condition for (18) needs to have $e^{ \pm i s}$, terms in coefficients of the rhs (righthand side) of (18) should be equal to zero, so that there are no secular terms. This leads to the condition

$$
\tau \mu_{2}-\frac{8 \tau \mu_{2} z_{s}}{3 \pi}\left(\alpha+\beta e^{-i \omega_{0} \tau}\right)+\frac{8 i \beta \omega_{2} \tau \mu_{0}}{3 \pi}\left(z_{s} e^{-i \omega_{0} \tau}\right)-i \omega_{2}=0,
$$

which can be divided into the two parts (real and imaginary) as follows:

$$
\begin{aligned}
& \tau \mu_{2}-\frac{8 \tau \mu_{2} z_{s}}{3 \pi}\left(\alpha+\beta \cos \left(\omega_{0} \tau\right)\right)+\frac{8 \beta \omega_{2} \tau \mu_{0}}{3 \pi}\left(z_{s} \sin \left(\omega_{0} \tau\right)\right)=0, \\
& \frac{8 \tau \beta \mu_{2} z_{s}}{3 \pi} \sin \left(\omega_{0} \tau\right)+\frac{8 \beta \omega_{2} \tau \mu_{0}}{3 \pi}\left(z_{s} \cos \left(\omega_{0} \tau\right)\right)-\omega_{2}=0 .
\end{aligned}
$$

This refers to two equations for $\mu_{2}$ and $\omega_{2}$, where $\omega_{0}, \mu_{0}$, and $z_{s}$ are non-negative solutions (positive solutions).

Figure 11 displays the corrections $\mu_{2}$ and $\omega_{2}$ for the one-term and two-term solutions. At $\tau=5$, the Hopf bifurcation point is established at $\left(\mu_{0}, \omega_{0}, z_{s}\right)=(1.90,2.37,0.60)$, where other positive values are $(D, \alpha, \beta)=(0.3,0.5,0.7)$. Solving (20) and (17) gives

$$
A=13.10 \times 10^{-1}+5.85 \times 10^{-1} i, \quad \mu_{2}=2.41, \quad \omega_{2}=-1.17 \times 10^{-2} .
$$

Using (10), we show leading-order approaches for the limit-cycle outcomes. The period will be

$$
\begin{aligned}
& u_{1}(s) \simeq z_{s}+\epsilon 2 \cos (\omega t), \quad \epsilon \simeq \sqrt{4.15 \times 10^{-1} \mu-7.88 \times 10^{-1}}, \\
& \omega \simeq 2.38-7.05 \times 10^{-3} \mu .
\end{aligned}
$$




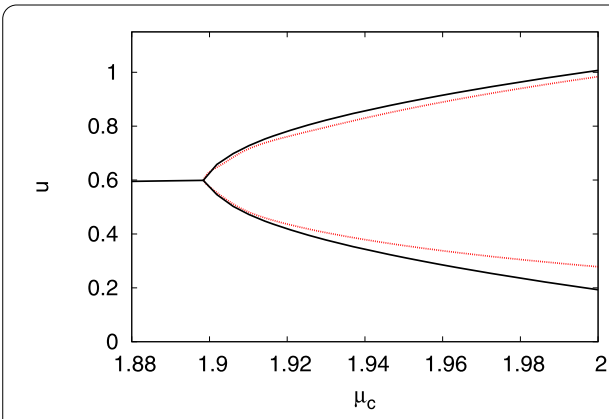

(a)

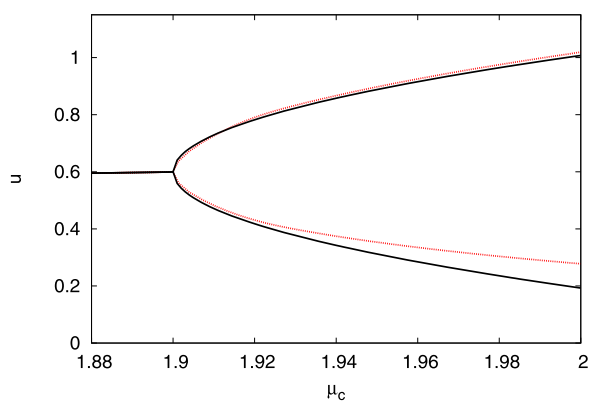

(b)

Figure 11 (color online) Bifurcation diagram of the population density in the $\mu-u$ map. The semi-analytical solutions (red dotted curve) and perturbation solutions (black solid curve) are obtained

The oscillation extrema are established while $\cos (\omega t)=\mp 1$, derived by $z_{s} \pm 2 \epsilon$. Here we mention that at large values of $\mu$ the amplitude of periodic solutions is increasing like $\mu^{1 / 2}$.

\subsection{Two-term semi-analytical solution}

The ODEs model for the semi-analytical two-term equations has been derived in Sect. 2. The method for deriving periodic results close to the point of Hopf bifurcation is similar to the case of one-term solutions outcome with additional modifications. We perform a form of solution (4) as follows:

$$
\begin{aligned}
& u_{1}(s)=z_{s 1}+\epsilon z_{1}(s)+\epsilon^{2} z_{2}(s)+\epsilon^{3} z_{3}(s)+\cdots \\
& u_{2}(s)=z_{s 2}+\epsilon z_{11}(s)+\epsilon^{2} z_{22}(s)+\epsilon^{3} z_{33}(s)+\cdots
\end{aligned}
$$

We substitute (23) and (10) into (4) and show equations at first three orders equation of $s$. Note that two equations are displayed at each step of $\epsilon$. Thus, at $\mathrm{O}(\epsilon)$ the solution at this case can be found as follows:

$$
z_{1}(s)=e^{i s}+\text { c.c. }, \quad z_{11}(s)=B e^{i s}+\text { c.c. }
$$

In order to explore the secondary oscillation amplitude related to $u_{2}$, we put (24) into the condition of $\mathrm{O}(\epsilon)$. We point out that $B$ is $u_{2}$ secondary complex oscillation, whose phase is not equal to the primary $u_{1}$-oscillation. The condition of $\mathrm{O}\left(\epsilon^{2}\right)$ shows the following outcome:

$$
z_{2}(s)=C_{1} e^{2 i s}+\text { c.c., } \quad z_{22}(s)=C_{2} e^{2 i s}+\text { c.c. }
$$

Putting (24) along with (25) in the $\mathrm{O}\left(\epsilon^{2}\right)$ condition, two complex expressions are found for amplitudes $C_{1}$ and $C_{2}$.

At $\mathrm{O}\left(\epsilon^{3}\right)$ the coupled equations are not presented here, due to their length. Both coupled equations include $e^{ \pm i s}$ in rhs, and this indicates the presence of secular terms. The solution $\left(z_{3}, z_{33}\right)=\Gamma e^{i s}(1, A)^{T}$ of the coupled system is a result of the homogeneous equation and therefore cannot be used to exclude secular terms at third order of $\epsilon\left(\mathrm{O}\left(\epsilon^{3}\right)\right)$. However, a choice orthogonal to this, such as $\left(z_{3}, z_{33}\right)=\Gamma e^{i s}(1,0)^{T}$, does not represent a homogeneous equation solution. So, secular terms can be eliminated by a corresponding selection of $\Gamma$, 
$\mu_{2}$, and $\omega_{2}$ values at the third-order level. For more details about finding $\mathrm{O}\left(\epsilon^{2}\right)$ and $\mathrm{O}\left(\epsilon^{3}\right)$, please see $[8,9,14]$. Again, we give a special example when $\tau=5$ to compute our results, so in this case the Hopf bifurcation happens at $\left(\mu_{0}, \omega_{0}, z_{s 1}, z_{s 2}\right)=(1.901,2.374,0.604,-0.007)$. Hence, solving equations at $\mathrm{O}\left(\epsilon^{1}\right), \mathrm{O}\left(\epsilon^{2}\right)$ and the secularity condition implies the following:

$$
\begin{aligned}
& B=3.11 \times 10^{-3}+1.21 \times 10^{-3} i=5.83 \times 10^{-3} e^{0.268 i}, \\
& C_{1}=3.23 \times 10^{-3}+4.02 \times 10^{-3} i, \quad C_{2}=2.05 \times 10^{-3}+7.13 \times 10^{-3} i, \\
& \mu_{2}=2.56, \quad \omega_{2}=-2.37 \times 10^{-2} .
\end{aligned}
$$

Therefore, the oscillatory result is displayed by

$$
\begin{aligned}
& u(s) \simeq z_{s 1}+z_{s 2}+2 \epsilon\left(\cos (w t)+\Re\left\{A e^{i s}+\bar{A} e^{-i s}\right\}\right), \\
& \epsilon \simeq \sqrt{3.91 \times 10^{-1} \mu-7.42 \times 10^{-1}}, \quad \omega \simeq 2.39-9.26 \times 10^{-3} \mu,
\end{aligned}
$$

where the extrema of the oscillatory outcome are $0.597 \pm 1.01 \epsilon$.

Figures 11(a) and 11(b) provide the bifurcation diagram of the $\mu$ values (the proliferation rate) against concentration $u$. The parameters are used here $\mu_{0}=1.9, D=0.3, \alpha=0.5$, $\beta=0.7$, and $\tau=5$. Both numerical perturbation (solid curve) and the results for the semianalytical solutions (dotted curve) are obtained. At Hopf supercritical bifurcation point, the solution becomes unstable, which occurs at $\mu_{c}=1.897$ for one-term case and at $\mu_{c}=$ 1.901 for the two-term case. The regions where periodic solutions have extrema oscillation amplitudes have been found. The periodic solution branches create an approximately right angle with the branch of steady-state solutions. The deviation from the exactly right angle is due to the fact that a finite number of points are plotted on the figure. Also, it can be seen that the comparison is better for the maximum amplitude than for the minimum amplitude for both one- and two-term numerical perturbations. Perturbation solutions and one-term semi-analytical solutions have an outstanding comparison for the interval $\mu_{c}<\mu<1.921$ as in Fig. 11(a). In addition, Fig. 11(b) shows perfect comparison between these solutions in the interval $\mu_{c}<\mu<1.928$, which are close to a point of Hopf bifurcation in both cases. It should be noted that the perturbation solution with limit-cycle is only a satisfactory outcome approximation in a limited $\mu$ range. The happens because periodic oscillation capacity grows rapidly when values of $\mu$ are increasing.

\section{Conclusions}

This paper derived the diffusive logistic equation with both mixed instantaneous and delayed density. The 1-D domain was investigated. Galerkin's method was exhibited to obtain a delay system of ODEs. We successfully performed the stability analysis, determined Hopf bifurcation regions, and constructed bifurcation maps. The effect of diffusion and delay values was fully discussed. We obtained that delay and diffusion values can influence the stability of regions of a parameter space. Consequently, as parameter values of delay increased, the values of the growth rate decreased. However, the diffusion values increased as the values of delay increased. Results in this paper were confirmed by contracted numerical examples and plotted stable and unstable limit cycles. No chaotic solutions were found, no period doubling was found in this model over a long period of time. 
In addition, an asymptotic method for the periodic outcome after the point of Hopf bifurcation was comprehensively discussed. Outcomes of numerical PDE model and semianalytical model supported the efficiency of the semi-analytical technique. It indicated that this technique can be helpful to present a precise analytical method for estimation of a PDEs system. In the future, we are looking forward to using this technique with another delay model with reaction-diffusion domain.

\author{
Acknowledgements \\ The author wishes to thank the anonymous referees and editor for their useful comments. \\ Funding \\ Not applicable. \\ Availability of data and materials \\ Not applicable. \\ Competing interests \\ The author declares that there is no conflict of interests regarding the publication of this paper
}

\title{
Authors' contributions
}

The author carried out the proofs of the main results and approved the final manuscript.

\section{Authors' information}

Associate Professor. H.Y. Alfifi, Basic Science Department, Imam Abdulrahman Bin Faisal University, Saudi Arabia.

\section{Publisher's Note}

Springer Nature remains neutral with regard to jurisdictional claims in published maps and institutional affiliations.

Received: 13 January 2020 Accepted: 31 March 2020 Published online: 17 April 2020

\section{References}

1. Al Noufaey, K.S., Marchant, T.R.: Semi-analytical solutions for the reversible Selkov model with feedback delay. Appl. Math. Comput. 232, 49-59 (2014)

2. Al Noufaey, K.S., Marchant, T.R., Edwards, M.P.: The diffusive Lotka-Volterra predator-prey system with delay. Math. Biosci. 270, 30-40 (2015)

3. Alfifi, H.Y. Semi-analytical solutions for the Brusselator reaction-diffusion model. ANZIAM J. 59, 167-182 (2017)

4. Alfifi, H.Y: Semi-analytical solutions for the delayed diffusive food-limited model. In: 2017 7th International Conference on Modeling, Simulation, and Applied Optimization (ICMSAO), Sharjah, United Arab Emirates, pp. 1-5 (2017)

5. Alfifi, H.Y: Semi-analytical solutions for the delayed and diffusive viral infection model with logistic growth. J. Nonlinear Sci. Appl. 12, 589-601 (2019)

6. Alfifi, H.Y., Marchant, T.R.: Feedback control for a diffusive delay logistic equation: semi-analytical solutions. IAENG Int. J. Appl. Math. 48(4), 317-323 (2018)

7. Alfifi, H.Y., Marchant, T.R., Nelson, M.I.: Generalised diffusive delay logistic equations: semi-analytical solutions. Dyn Contin. Discrete Impuls. Syst., Ser. B, Appl. Algorithms 19, 579-596 (2012)

8. Alfifi, H.Y., Marchant, T.R., Nelson, M.I.: Semi-analytical solutions for the 1- and 2-D diffusive Nicholson's blowflies equation. IMA J. Appl. Math. 79, 175-199 (2014)

9. Alfifi, H.Y., Marchant, T.R., Nelson, M.I.: Non-smooth feedback control for Belousov-Zhabotinskii reaction-diffusion equations: semi-analytical solutions. J. Math. Chem. 57, 157-178 (2016)

10. Alharthi, M.R., Marchant, T.R., Nelson, M.l.: Mixed quadratic-cubic autocatalytic reaction-diffusion equations: semi-analytical solutions. Appl. Math. Model. 38, 5160-5173 (2014)

11. Busenberg, S., Huang, W.: Stability and Hopf bifurcation for a population delay model with diffusion effects. J. Differ. Equ. 124(1), 80-107 (1996)

12. Chen, S., Shi, J.: Stability and Hopf bifurcation in a diffusive logistic population model with nonlocal delay effect. J. Differ. Equ. 12, 3440-3470 (2012)

13. Chiu, K.S., Li, T.: Oscillatory and periodic solutions of differential equations with piecewise constant generalized mixed arguments. Math. Nachr. 292, 2153-2164 (2019)

14. Erneux, T:: Applied Delay Differential Equations. Springer, New York (2009)

15. Feng, W., Lu, X.: On diffusive population models with toxicants and time delays. J. Math. Anal. Appl. 233, 374-386 (1999)

16. Friesecke, G.: Convergence to equilibrium for delay-diffusion equations with small delay. Math. Biol. 5(1), 89-103 (1993)

17. Gourley, S.A., Ruan, S.: Dynamics of the diffusive Nicholson's blowflies equation. Proc. R. Soc. Edinb., Sect. A 130A, 1275-1291 (2000)

18. Hale, J.: Theory of Functional Differential Equations. Springer, New York (1977)

19. Li, T., Pintus, N., Viglialoro, G.: Properties of solutions to porous medium problems with different sources and boundary conditions. Z. Angew. Math. Phys. 70, 1-18 (2019) 
20. Li, T., Viglialoro, G.: Analysis and explicit solvability of degenerate tensorial problems. Bound. Value Probl. 2018, Article ID 2 (2018)

21. Looss, G., Joseph, D.D.: Elementary Stability and Bifurcation Theory, 2nd edn. Springer, New York (1990)

22. Manna, K.: A non-standard finite difference scheme for a diffusive HBV infection model with capsids and time delay. J. Differ. Equ. Appl. 23(11), 1901-1911 (2017)

23. Marchant, T.R.: Cubic autocatalytic reaction diffusion equations: semi-analytical solutions. Proc. R. Soc. Lond. A 458, 873-888 (2002)

24. Marchant, T.R., Nelson, M.I.: Semi-analytical solution for one-and two-dimensional pellet problems. Proc. R. Soc. Lond. A 460, 2381-2394 (2004)

25. Miao, H., Teng, Z., Abdurahman, X., Li, Z.: Global stability of a diffusive and delayed virus infection model with general incidence function and adaptive immune response. Comput. Appl. Math. 37(3), 3780-3805 (2018)

26. Nicholson, A.J.: An outline of the dynamics of animal populations. Aust. J. Zool. 2, 9-65 (1954)

27. Shah, R., Li, T.: The thermal and laminar boundary layer flow over prolate and oblate spheroids. Int. J. Heat Mass Transf. 121, 607-619 (2018)

28. Shi, J., Shivaji, R.J.: Persistence in reaction diffusion models with weak Allee effect. Math. Biol. 52(6), 807-829 (2006)

29. Smith, G.D.: Numerical Solution of Partial Differential Equations: Finite Difference Methods, 3rd edn. Oxford University Press, New York (1985)

30. So, J.W.-H., Wu, J., Yang, Y.: Numerical steady state and Hopf bifurcation analysis on diffusive Nicholson's blowflies equation. Appl. Math. Comput. 111, 33-51 (2000)

31. Su, Y., Wei, J., Shi, J.: Hopf bifurcations in a reaction diffusion population model with delay effect. J. Differ. Equ. 247, 1156-1184 (2009)

32. Su, Y., Wei, J., Shi, J.: Hopf bifurcation in a diffusive logistic equation with mixed delayed and instantaneous density dependence. J. Dyn. Differ. Equ. 24, 897-925 (2012)

33. Vales, E., Gerardo, G.: Analysis of a HBV model with diffusion and time delay. J. Appl. Math. 2012, 1-25 (2012)

\section{Submit your manuscript to a SpringerOpen ${ }^{\circ}$ journal and benefit from:}

- Convenient online submission

- Rigorous peer review

- Open access: articles freely available online

- High visibility within the field

- Retaining the copyright to your article

Submit your next manuscript at $>$ springeropen.com 